\title{
3D Reconstruction of Mitochondrial Complex I Analyzed After Biogenesis in the Absence of Assembly Factor N7BML (NDUFAF2)
}

\author{
Christine T. Nolan ${ }^{1}$, Katarzyna Kmita ${ }^{2}$, Volker Zickermann², Teresa Ruiz ${ }^{1}$, Michael Radermacher ${ }^{1}$ \\ 1. Department of Molecular Physiology and Biophysics, University of Vermont, Burlington VT, USA \\ 2. Institute of Biochemistry II, Medical School, Goethe University, Frankfurt/M, Germany
}

Complex 1(NADH:ubiquinone oxidoreductase) is the first and largest electron transport chain enzyme in bacteria and in the inner membrane of mitochondria. Complex 1 consists of two arms forming an "L" shape. One arm lies in the inner membrane of the mitochondria, while the other protrudes out into the mitochondrial matrix. After the matrix arm binds NADH, two electrons are transferred via a series of iron-sulfur clusters to ubiquinone with concomitant translocation of four protons across the membrane. The reduced ubiquinone is then transferred to complex III, cytochrome $\mathrm{C}$ is reduced and further processed in complex IV. In the process of oxidizing NADH, a total of 10 electrons are translocated across the membrane, creating a membrane potential that powers ATP production by complex V.

The minimal bacterial complex I contains 14 subunits that are preserved through all species. Eukaryotic complex 1 has more than 21 additional (accessory) subunits and possesses a molecular weight of approximately $1 \mathrm{MDa}$. The function of a large number of accessory subunits is still unknown. Complex I assembly is a multi-step process supported by assembly factors that transiently bind to subassemblies of the enzyme [1]. At least five assembly factors are involved in the biogenesis of eukaryotic complex I. NDUFAF2 is involved in the late stages of complex I assembly, specifically in the final assembly of the matrix arm, although this factor is not absolutely essential for the process. The absence of NDUFAF2 seems not to affect the ubiquinone reductase activity of the assembled complex I, however, in $N$. crassa mitochondria an increase of the production of reactive oxygen species has been reported [2].

N7BML is a yeast ortholog of NDUFAF2 [3]. We have analyzed purified complex I from the yeast Yarrowia lipolytica, lacking assembly N7BML $(\mathrm{nb} 7 \mathrm{ml} \Delta)$. Mitochondrial extracts showed significantly decreased complex I content, but the purified complex showed a ubiquinone reductase activity comparable to wild type [3]. All subunits were present in the assembled complex as determined by dSDS PAGE and proteomic analysis [3].

Structural studies were performed by 3D electron microscopy of single particles, using the random conical tilt reconstruction technique. The sample was applied to carbon coated grids at a concentration of $0.0015 \mathrm{mg} / \mathrm{ml}$ and stain embedded with $2 \%$ PTA. Pairs of images were recorded with a nominal magnification of $52 \mathrm{kX}$ and tilt angles of approximately $55^{\circ}$ and $0^{\circ}$ using a $100 \mathrm{kV}$ accelerating voltage. Images were scanned on a SCAI flatbed scanner with $7 \mu \mathrm{m}$ pixel size, then binned by a factor of 3 , which resulted in a $4.02 \AA$ final pixel size at the specimen scale. Image processing was carried out using SPIDER version 5.0 with extensions. A total of 9815 pairs of particles were selected. The $0^{\circ}$ images were processed first by a single-reference alignment followed by correspondence analysis. This process was continued with three iterations of multi-reference alignment, each followed by correspondence analysis and classification. Nine final classes were obtained, and nine 3D reconstructions were calculated from the corresponding tilt images. The 3D reconstructions were refined using 3D reference based projection alignments based on Radon transforms [4]. 
Five of the reconstructions $(1-4,6)$ closely resemble the wild type enzyme, with small conformational variations and differences in orientation. Classes 7-9 have low occupancy and are consequently not well defined. Class 5 shows a mixture of particles that need further analysis. At the current resolution no significant structural differences can be observed between the mutant and wild type complex I [5], indicating that the lack of assembly factor 2 does not introduce major structural changes. Our findings are consistent with the observation that $n b 7 \mathrm{ml} \Delta$ complex I was completely assembled and showed close to normal ubiquinone reductase activity [3]. Higher resolution might still reveal small structural differences that could affect the stability or regulation of complex I without affecting its basic function.

\section{References:}

[1] McKenzie M, Ryan MT, IUBMB Life 62 (2010), p497

[2] Pereira B., Videira A., Duarte M., Mol. Cell. Biol. 33 (2013), p2623

[3] Kmita et al., submitted

[4] Radermacher M., Scanning Microscopy 11 (1997), p171

[5] Radermacher M. et al. J Struct Biol. 154(3) (2006), p269.

[6] The authors acknowledge funding from NIH, grant 2RO1 GM068650 to M.R, and from DFG grants ZI 552/3-1 to V.Z. and EXC 115 to V.Z.
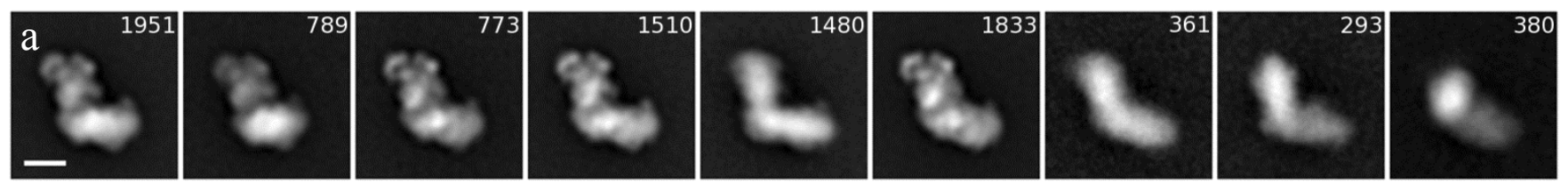

b
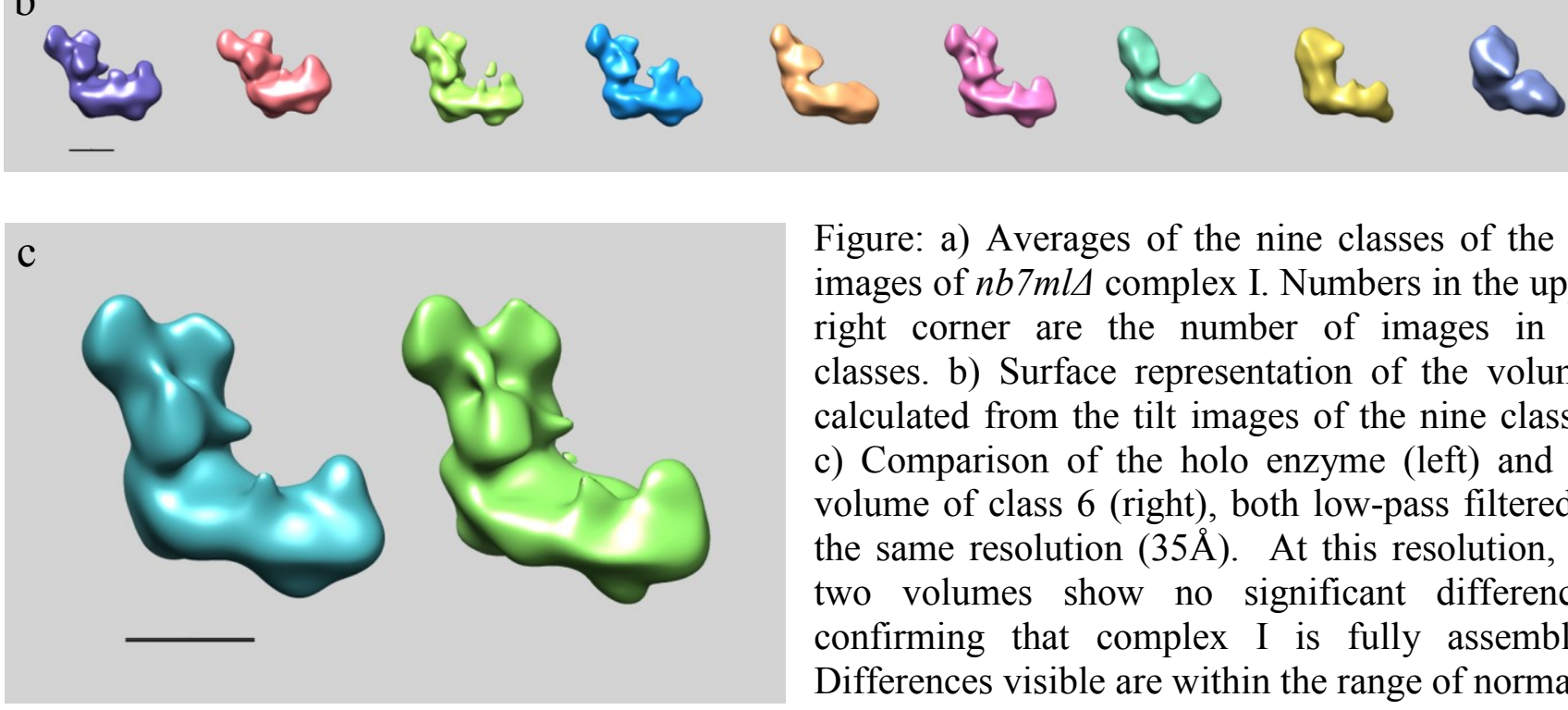

Figure: a) Averages of the nine classes of the $0^{\circ}$ images of $n b 7 \mathrm{ml} \Delta$ complex I. Numbers in the upper right corner are the number of images in the classes. b) Surface representation of the volumes calculated from the tilt images of the nine classes. c) Comparison of the holo enzyme (left) and the volume of class 6 (right), both low-pass filtered to the same resolution $(35 \AA)$. At this resolution, the two volumes show no significant differences, confirming that complex I is fully assembled. Differences visible are within the range of normal flexibility observed in samples of the complex. Scale bars $10 \mathrm{~nm}$. 